\title{
Permanent Night Work in Germany
}

\author{
Corinna BRAUNER', Grit MÜLLER, Anne M. WÖHRMANN
}

Bundesanstalt für Arbeitsschutz und Arbeitsmedizin (BAuA)

\begin{abstract}
Research on night work focuses almost solely on night work as part of rotating shift schedules. Thus, little is known about permanent night workers, their working conditions and health. The aim of this study is to give insight on characteristics of permanent night workers, their health status and their work-life balance. Data from the BAuA-Working Time Survey 2015 were used and 189 employees in permanent night work identified. Results are presented in comparison to statistics on employees in general and to rotating shift workers with night shifts in particular. The most common occupations among permanent night workers are professional nurses and heavy truck and lorry drivers. Night workers less often rated their health as (very) good or were satisfied with their work-life balance compared to employees in general. They also reported health complaints more often. Findings in comparison to employees working in rotating shifts with night work were mixed. The results point towards some concerns regarding health and work-life balance related to permanent night work.
\end{abstract}

Keywords: health, night shift, working time, work-life balance, working conditions

\section{Introduction}

Permanent night work is a specific type of shift work in which employees work solely or almost solely at night (Arlinghaus et al. 2016). Working at night can affect employees' health and well-being due to disruption in circadian rhythms and the sleep-wake-cycle as well as reduced possibilities for social contacts (Tucker/Folkard 2012). Nevertheless, permanent night work cannot be completely avoided. Our society in general and companies in particular rely on night work for demand-oriented, technological or economic reasons (Hellert 2014). Night work for demand-oriented reasons includes all services that ensure the provision of care and safety as well as power supply. Night work for technological reasons is necessary in those industries where manufacturing processes take longer than a regular work day, where manufacturing facilities cannot be turned off or where biological processes are involved that must be

${ }^{1}$ All three authors are researchers at the Federal Institute for Occupational Safety and Health (BAuA) based in Dortmund, Germany. Corinna Brauner's research focuses on working time and the work-home interface. Grit Müller is head of a project examining the consequences of permanent night work and 12-hour shifts. Anne M. Wöhrmann is project head of the BAuA Working Time Survey. Corresponding author: Corinna Brauner: brauner.corinna@baua.bund.de. 
monitored overnight. Economic reasons come into play if companies want to ensure maximum economic utilization of manufacturing facilities and want to be able to adjust their production according to demands (Hellert 2014). For employees, reasons to work in permanent night shifts are diverse. Important incentives are probably the higher income and other economic rewards that are earned with night shifts. Other reasons may be that one's occupation is usually performed at night, that job demands are lower or different in night shifts or that permanent night work provides a possibility to work in non-rotating shifts or to ensure a better compatibility between work, leisure and family (Arlinghaus et al. 2016).

Current statistics show that 19\% of European employees (Eurofound 2017) and around 6\% of female and $12 \%$ of male German employees (Bundesanstalt für Arbeitsschutz und Arbeitsmedizin 2017) were engaged in night work as part of rotating shifts or permanent night work in 2015 and 2016, respectively. For Germany, these numbers have been slightly but steadily increasing over the last two decades: Estimates based on the German Microcensus show that the number of night workers has increased by nearly $2 \%$ in women and over $3 \%$ in men since 1996 (Pfahl et al. 2017). The German Federal Statistical Office estimated a number of 700,000 permanent night workers in Germany in 2009 (Puch 2010). Despite the increasing relevance of night work, figures and studies that focus explicitly on permanent night work are rare. For instance, little is known about which employees work in permanent night shift, which industries and occupations they work in and how their working (time) conditions look like.

Further, due to the special demands night work poses to employees, permanent night work is also an important concern in occupational health and safety research. Potential mechanisms linking night work to poor health or increased accident risk are diverse. In their conceptual model on abnormal work schedules, Tucker and Folkard (2012) outlined that work schedule features may lead to a disturbed body clock, shortened and disturbed sleep as well as disturbed family and social life. These adverse consequences of abnormal work schedules may be directly associated with acute reactions such as fatigue, impaired mood or lower performance but also with chronic mental health problems and lasting decreases in performance. The model predicts that ultimately, these acute and chronic effects may translate into health and safety issues. Tucker and Folkard (2012) point out that night work conflicts with the normal sleepwake cycle, and thus it is often associated with a disruption of circadian rhythms similar to jetlag. Since exposure to sunlight prevents the onset of melatonin - a sleep hormone - night workers also face difficulties when trying to fall asleep during the day or staying awake during the night (Eastman et al. 1994; Czeisler/Dijk 1995). However, even the body clocks of permanent night workers with several consecutive night shifts are rarely completely adjusted (Folkard 2008) because during free days most permanent night workers return to their dayoriented life. On work days, however, night workers often sleep when others are awake. Thus, night workers also encounter greater challenges in maintaining a normal family and social life.

Studies - most of them dealing with night work as a part of rotating shifts - point to a number of health and safety issues related to night work: It is associated with a variety of physical and mental health problems, including chronic conditions like type 2 diabetes (Knutsson/Kempe 2014), depression (Angerer et al. 2017), insomnia, fatigue (Øyane et al. 2013) and health complaints (Alfredsson et al. 1991). Night work has also been found to be 
associated with adverse health behaviors such as smoking, calorie and caffeine intake and thus an increased risk for cardiovascular problems and cancer (Ramin et al. 2015). Further, data on occupational accidents in Germany show that shift workers and especially night workers experience occupational accidents more often than workers not working in shifts (Liersch 2014).

Unfortunately, there is a lack of studies focusing explicitly on permanent night work as the shiftwork literature is mostly dominated by studies on employees working in rotating shifts. Further, many studies do not distinguish between permanent night workers and those in rotating shifts. This gap in the literature slows down advances in the improvement of working conditions of permanent night workers and shift workers in rotating shift systems alike.

With the present study, we aim to provide insights on the group of permanent night workers. We present descriptive results on permanent night workers from the BAuA-Working Time Survey 2015 - a study that is representative for large parts of the German working population. These results are given together with statistics on employees in general as well as on shift workers in rotating shift systems with night shifts ("rotating night shift workers"). More specifically, we focus on the distribution of permanent night work within the workforce, socio-demographic characteristics, job and working time characteristics. Further, we put a special emphasis on permanent night workers' health and work-life balance. Thereby, we provide a more holistic picture and contribute to a better understanding of permanent night work in Germany.

\section{BAuA-Working Time Survey 2015}

For the current study, data from the BAuA-Working Time Survey 2015 were used. This is a representative survey of 20,000 working persons in Germany (with a weekly working time of at least 10 hours). Data collection took place in 2015. Applying a dual-frame-approach (calls via random landline and mobile numbers), computer-assisted telephone interviews with a mean duration of 35 minutes were conducted. Participants were interviewed by trained interviewers. Amongst others, the participants were asked questions on different topics of working time, other working conditions as well as health aspects. More detailed information is given in the methods report of the BAuA-Working Time Survey 2015 (Häring et al. 2016). For the present study, employees aged 15 to 65 years were eligible.

The subsample of persons working in permanent night shift was identified in two ways: First, in the interview, participants were asked whether they normally worked between 7 am and $7 \mathrm{pm}$. Employees who indicated that their usual working times are not within the time frame from $7 \mathrm{am}$ to $7 \mathrm{pm}$ were asked whether they worked in shifts. If so, they were asked if they worked in permanent night shifts $(N=69)$. Second, all employees who indicated not working between $7 \mathrm{am}$ and $7 \mathrm{pm}$ but negated working in shifts were screened for additional permanent night workers $(N=120)$. To do so, the participants' specifications regarding the starting and ending times of their usual working day were screened. In line with the night work definition in the German Working Time Act (Bundesministerium für Arbeit und Soziales 2017), employees were included in the sample if they worked at least 2 hours between $11 \mathrm{pm}$ and $6 \mathrm{am}$ (for bakers and confectioners: 2 hours between $10 \mathrm{pm}$ and $5 \mathrm{am}$ ). Occupations of these employees were checked on a case-by-case basis to validate status as night work- 
ers. Thus, the final sample consisted of $N=189$ permanent night workers. Because of the posthoc identification and the survey's filtering procedures only permanent night workers who affirmed working in shifts received all questions regarding shift and night work. Thus, some analyses could only be conducted for a reduced sample.

More information on the sample characteristics is given in the next sections. For permanent night workers, we present unweighted data due to the small sample size. Further, we report comparable values using weighted data of rotating night shift workers who indicated that they worked in a rotating shift system which includes night shifts $(N=1,156)$. Where meaningful, we also present values for all (dependent) employees aged 15 to 65 years based on weighted data $(N=18,119)$ as a reference. The latter are mostly based on the BAuA-Working Time Report 2016 (Wöhrmann et al. 2016).

\section{Socio-demographic Characteristics}

Socio-demographic characteristics of permanent night workers, rotating night shift workers, and employees in Germany in general are displayed in Table 1. Slightly more permanent night workers are male than female. This proportion is similar to the gender distribution among the total sample while the percentage of males is higher among rotating night shift workers. The average permanent night worker is four years older than the average rotating night shift worker and three years older than the average employee. Differences also arise in terms of the level of education. This was assessed using the International Standard Classification of Education 2011 (UNESCO Institute for Statistics ISCED 2011). The vast majority of permanent night workers and rotating night shift workers has graduated from school or has received vocational training. Thus, a medium level of education is more common for permanent night workers and rotating night shift workers than employees in general. However, permanent night workers are less likely to hold an academic degree or a master craftman's diploma than rotating night shift workers or employees in general. In addition, permanent night workers are slightly more likely to have no school-leaving qualification. These findings are in accord with those presented by the German Federal Statistical Office on night workers in permanent and rotating night shifts (Puch 2010).

Regarding the life situation, our analyses show that the percentage of permanent night workers and rotating night shift workers living with their partner or spouse is smaller than among employees in general. Nearly one in three permanent night workers and rotating night shift workers indicated that children under 18 years lived in their household. Thus, permanent night workers and rotating night shift workers are slightly less likely to be living in a household with children compared to employees in Germany in general. 
Table 1: Socio-demographic Characteristics of Permanent Night Workers, Rotating Night Shift Workers, and Employees in General

\begin{tabular}{llll}
\hline & Permanent night workers & Rotating night shift workers & All employees \\
\hline Gender & $54.5 \%$ male & $65.9 \%$ male & $53.1 \%$ male \\
Age & $45.5 \%$ female & $31.1 \%$ female & $46.9 \%$ female \\
& $M=45.8$ years & $M=41.7$ years & $M=42.9$ years \\
& $S D=11.6$ years & $S D=11.2$ years & $S D=11.6$ years \\
Education & Range = 19-64 years & Range = 19-65 years & Range = 16-65 years \\
& $6.9 \%$ low & $5.3 \%$ low & $4.8 \%$ low \\
Living with & $76.2 \%$ medium & $72.9 \%$ medium & $58.7 \%$ medium \\
partner or & $16.9 \%$ high & $21.8 \%$ high & $36.6 \%$ high \\
spouse & partner/spouse & $64.4 \%$ living with & $67.1 \%$ living with \\
& $40.4 \%$ living without & partner/spouse & partner/spouse \\
Children under & $31.9 \%$ with children & $35.6 \%$ living without & $32.9 \%$ living without \\
18 in the & $68.1 \%$ without children & partner/spouse & partner/spouse \\
household & & $31.4 \%$ with children & $34.0 \%$ with children \\
\hline
\end{tabular}

Note: Education: low = no school-leaving qualification, medium = school-leaving qualification or vocational training, high = academic degree or master craftman's diploma.

\section{Occupational and Job Characteristics}

Study participants were asked to indicate the economic sector of the company they work for (see Figure 1). More than one in four permanent night worker is employed in the service sector and one in five permanent night workers works in the public sector. Work in industry, craft or commerce is less common among permanent night workers. Compared to rotating night shift workers and employees in general, permanent night workers work noticeably more often in the service sector and less often in the public sector or industry.

Employees were further asked for a more detailed characterization of the industry that the company they work for is engaged in. Open answers were coded following the German Classification of Economic Activities (WZ 2008; German Federal Statistical Office 2008). Permanent night workers most commonly mentioned human health and social work activities (22.9\%; WZ 2008: Q) with the majority working in human health activities (WZ 2008: Q86). In second place, $17.1 \%$ of permanent night workers reported working in manufacturing (WZ 2008: C), mostly in the manufacturing of food products. Almost as many permanent night workers indicated working in transportation and storage (16.6\%; WZ 2008: H), mostly warehousing and support activities for transportation (WZ 2008: H52). This pattern of results is in line with analyses of the German Federal Statistical Office (Puch 2010) which found that night work in permanent or rotating shifts was most common among manufacturing industries, in the health care and public transport sector. 
Figure 1: Distribution of Economic Sectors among Permanent Night Workers, Rotating Night Shift Workers, and Employees in General

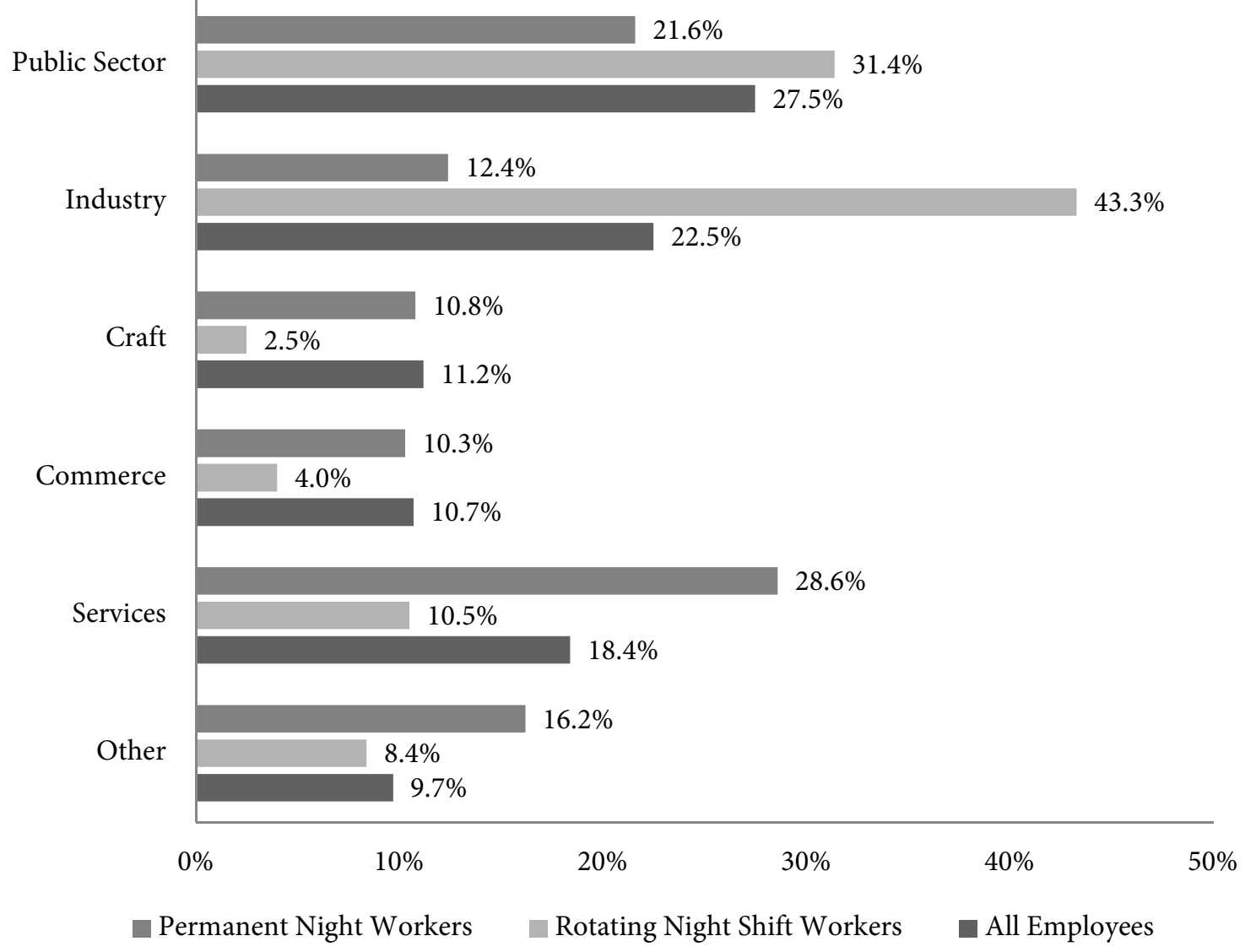

In our study, employees were also asked for a characterization of their profession. Open answers were categorized according to the International Standard Classification of Occupations 2008 (ISCO 2008; International Labour Office 2012). The three most common professions mentioned by permanent night workers correspond to the three most common industries: Permanent night workers were most often employed as health associate professionals (18.3\%; ISCO 2008: 32), and more specifically associate professional nurses (ISCO 2008: 3221). In second place were drivers and mobile plant operators (17.2\%; ISCO 2008: 83), and more specifically heavy truck and lorry drivers (ISCO 2008: 8332). In third place, permanent night workers reported a profession in food processing, woodworking, garment and other craft and related trades workers (10.2\%; ISCO 2008: 75), most of which were employed as bakers, pastry-cooks and confectionery makers (ISCO 2008: 7512).

Further, our analyses show that, in terms of company size, $36.0 \%$ of permanent night workers are employed in small companies with less than 50 employees. Almost as many permanent night workers (34.8\%) work in mid-sized companies with 50 to 250 employees; $29.2 \%$ are employed in large companies with more than 250 employees. Thus, compared to employees in Germany in general, permanent night workers more often work in mid-sized companies and less often in small or large companies. Rotating night shift workers, on the other hand, work considerably more often in large companies (59.0\%). 


\section{Working Conditions}

Previous research found that shift and night workers are more exposed to adverse psychosocial and physical working conditions (Beermann 2010). Therefore, we examined specific working conditions associated with permanent night work and shift work (see Table 2), some of which may pose a risk to employees' health. Note that many of these working conditions may be attributed primarily to the specific occupations in which permanent night work or shift work is most dominant and to a lesser extent to the time of day (or night) at which people work.

Table 2: Working Conditions of Permanent Night Workers, Rotating Night Shift Workers, and Employees in General

\begin{tabular}{|c|c|c|c|}
\hline & $\begin{array}{l}\text { Permanent night } \\
\text { workers }\end{array}$ & $\begin{array}{l}\text { Rotating night shift } \\
\text { workers }\end{array}$ & All employees \\
\hline Deadline or performance pressure & $52.4 \%$ & $58.4 \%$ & $51.2 \%$ \\
\hline Need to work very quickly & $58.8 \%$ & $59.6 \%$ & $48.8 \%$ \\
\hline Working in a standing position & $74.6 \%$ & $81.7 \%$ & $53.9 \%$ \\
\hline Lifting and carrying heavy loads & $47.1 \%$ & $39.6 \%$ & $23.5 \%$ \\
\hline $\begin{array}{l}\text { Working in a bent, squatting, } \\
\text { kneeling or recumbent position, } \\
\text { working overhead }\end{array}$ & $27.0 \%$ & $24.4 \%$ & $19.5 \%$ \\
\hline $\begin{array}{l}\text { Working exposed to cold, heat, } \\
\text { moisture, humidity or draughts }\end{array}$ & $42.3 \%$ & $40.3 \%$ & $24.7 \%$ \\
\hline $\begin{array}{l}\text { Working under bright light or in } \\
\text { light conditions that are poor or too } \\
\text { low }\end{array}$ & $33.5 \%$ & $26.2 \%$ & $11.9 \%$ \\
\hline Working exposed to noise & $29.1 \%$ & $56.0 \%$ & $28.4 \%$ \\
\hline
\end{tabular}

Note: Depicted is the percentage of participants who often experience these working conditions

In terms of psychological working conditions, a similar share of permanent night workers and all dependent employees reported frequent deadline or performance pressure while this percentage is slightly higher among rotating night shift workers. Both, permanent night workers and rotating night shift workers have to work very quickly more often than employees in general. For the most part, permanent night work and shift work is also physically demanding work. Permanent night workers and rotating night shift workers more often work in a standing position or in a bent, squatting, kneeling, recumbent or overhead position than employees in general. The share of people who often lift and carry heavy loads is highest among permanent night workers. Permanent night work and shift work is also often characterized by adverse ambient environmental conditions. In comparison to employees in general, permanent night workers and rotating night shift workers are more frequently exposed to cold, heat, moisture, humidity or draughts in their work. Moreover, permanent night workers also more often report working under unfavorable lighting conditions than rotating night shift workers or employees in general. Regarding the exposure to noise, permanent night shift workers do 
not differ from employees in general while exposure to noise is considerably higher among rotating night shift workers.

Other studies on night work found a similar pattern of results: Representative surveys showed that night workers were more often affected by adverse psychosocial demands, e.g. lack of decision latitude, monotone tasks, but especially physical demands, such as difficult body posture and heavy lifting, work with hazardous contaminants, noise and vibrancy exposure, and exposure chemicals, dust, smoke, or gas (Struck et al. 2012; Liersch 2014).

\section{Night Shift and Working Time Characteristics}

To further enhance our understanding about the work histories and working hours of permanent night workers, an overview of night shift characteristics is provided in Figure 2 . Note that some questions concerning aspects specific to night shift were only administered to employees who indicated during the interview that they worked in shifts. Questions on general aspects of working hours were answered by all participants.

On average, at the time of the interview, permanent night workers had been working in night shifts for 9.2 years $(S D=8.3$, range $=0-28)$. Half of them had been working in night shifts for a period of five or less years. One quarter of permanent night workers had been working in night shifts for a period of six to fifteen years. Another quarter had been working in night shifts for more than fifteen years. Overall, permanent night workers had been working in night shifts for a shorter time than rotating night shift workers. Nevertheless, every third permanent night worker had been working night shifts for at least half of his or her adult life. The average permanent night worker works 6.1 consecutive night shifts $(S D=5.5$, range $=$ 1-30). About three in ten permanent night workers work up to four consecutive night shifts. Most commonly, permanent night workers have five consecutive night shifts, particularly if they work in manufacturing/industry. About one in four permanent night workers works six or seven consecutive night shifts, and about one in ten permanent night workers works even more. According to expectations, rotating night shift workers have less consecutive night shifts than permanent night workers.

On average, permanent night workers work 16.8 nights per month $(S D=6.1$, range $=4$ 30). Roughly one in five permanent night workers work ten or less night shifts per month. The majority works eleven to 20 night shifts. About one in five permanent night workers works exactly 20 night shifts per month and just as many permanent night workers work more than 20 night shifts per month. Not surprisingly, rotating shift workers have fewer night shifts per month.

Permanent night workers work on average 38.5 hours per week $(S D=14.5$, range $=10$ 84). Almost one third (31.4\%) work in part time jobs with less than 35 actual weekly working hours. The share of part time workers is thus considerably higher than among rotating night shift workers (10.3\%) and employees in general (23.2\%). Most permanent night workers (45.9\%) have a full-time job with less than 48 weekly working hours. However, this share is lower than that of rotating night shift workers $(68.9 \%)$ and employees in general $(60.3 \%)$. More than one in five (22.7\%) permanent night workers work 48 hours or more in a normal 
week. Thus, overlong hours are slightly more common among permanent night workers than among rotating shift workers (20.8\%) and employees in general (16.5\%).

\section{Figure 2: Overview of Night Shift Characteristics}

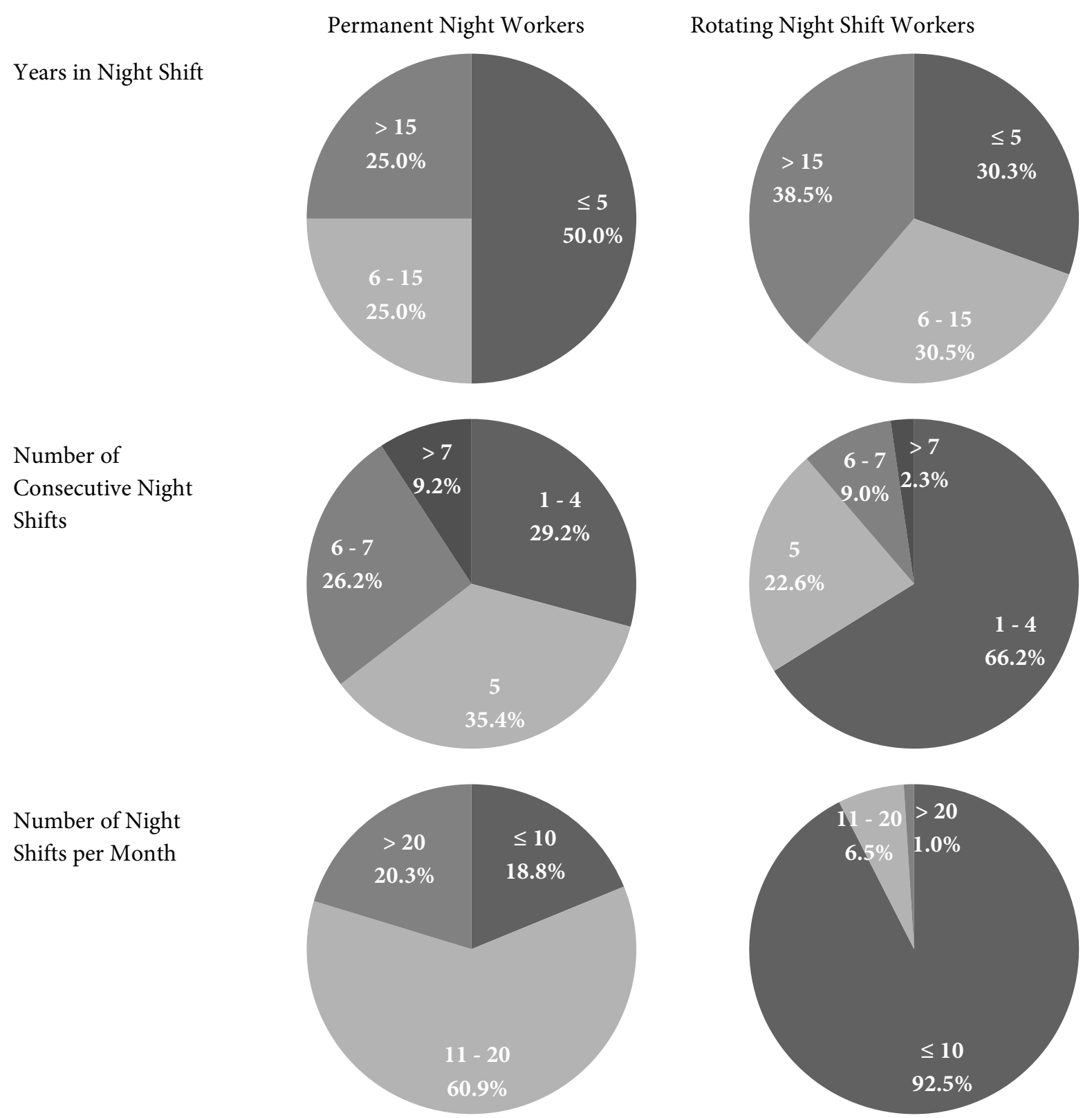

Weekend work is more common among permanent night workers (86.5\%) and rotating night shift workers (88.7\%) than among employees in general (43.4\%). While only $12.3 \%$ of rotating night shift workers work on Saturdays (at least once per month) but not on Sundays, this share is more similar among permanent night workers (22.1\%) and all employees (19.9\%). However, major differences can be found in relation to work on Sundays and holidays. While working on Sundays or holidays at least once a month is reported by almost two in three permanent night workers (64.4\%) and three in four rotating night shift workers (76.4\%), this is only the case for $23.5 \%$ of employees in Germany in general. 
Despite their atypical working hours, three out of four (76.2\%) permanent night workers are satisfied or very satisfied with their working hours. This is a slightly higher percentage than among rotating night shift workers $(71.4 \%)$ but lower than among employees in general (83.0\%).

\section{Work-life Balance and Health}

Working at night entails far-reaching consequences for employees' private and family lives (Tucker/Folkard 2012). Previous studies found that night workers are affected by work-family conflict to a greater extent than regular day workers (Beutell 2010). A similar picture became apparent in the present study: Permanent night workers seem to experience more difficulties in juggling the demands of work and private life than employees in Germany in general (see Figure 3). However, they seem to be slightly more often satisfied with the work-life balance than rotating night shift workers.

Figure 3: Work-life Balance and Subjective Health Status of Permanent Night Workers, Rotating Night Shift Workers, and Employees in General

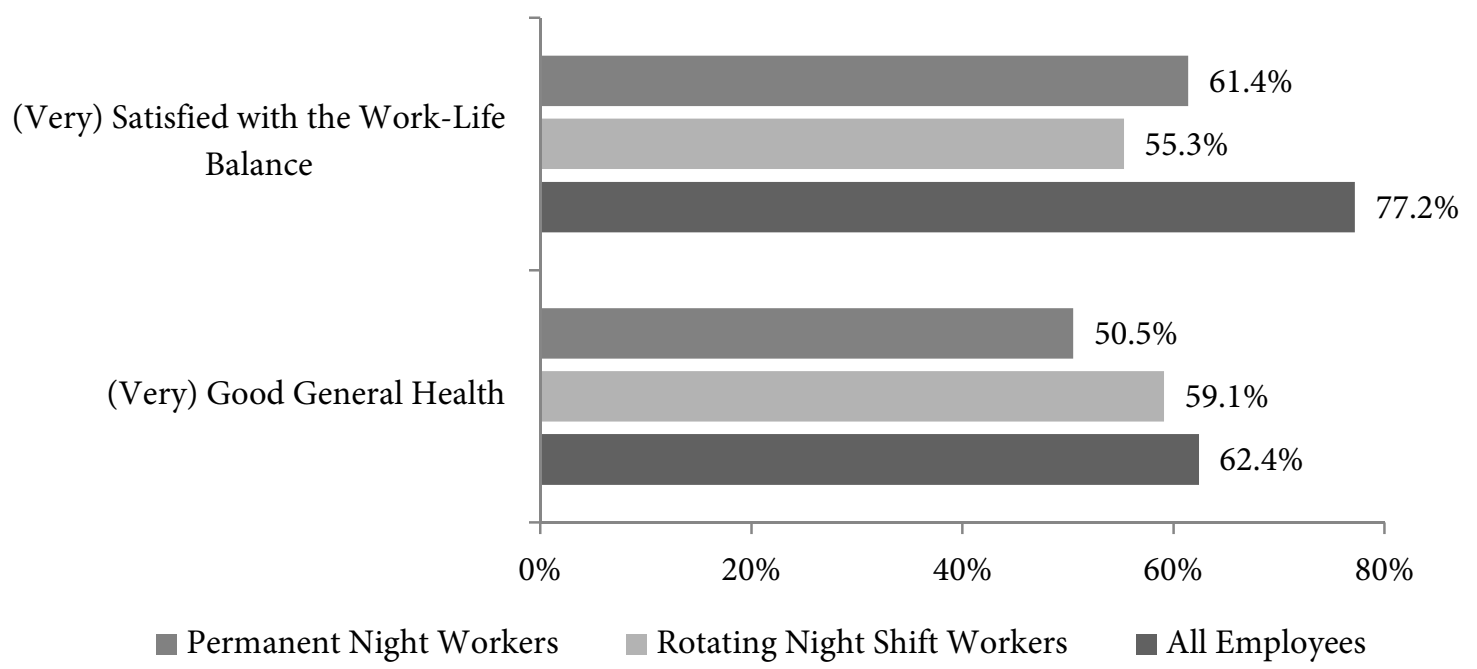

Satisfaction with the work-life balance is relatively similar for permanent night workers with (very satisfied/satisfied with their work-life balance: $61.7 \%$ ) or without (60.9\%) children and for permanent night workers living with $(63.4 \%)$ or without $(59.2 \%)$ a partner or spouse. However, in line with the results for employees in Germany in general as presented in the BAuA-Working Time Report 2016 (Wöhrmann et al. 2016), satisfaction with the work-life balance is dramatically lower in case of overlong working hours of 48 and more hours per week. Only $38.1 \%$ of permanent night workers with overlong working hours are (very) satisfied with the work-life balance compared to $60.0 \%$ of full-time workers who work less than 48 hours per week and $81.0 \%$ of part-time workers. Interestingly, satisfaction with the work-life balance is higher for permanent night workers who work at the weekend: $61.1 \%$ of those working on Saturdays (but not on Sundays) and 63.8\% of those working (also) on Sundays 
and holidays are (very) satisfied with the work-life balance compared to 50.0\% who do not regularly work at the weekend.

The model proposed by Tucker and Folkard (2012) on abnormal work schedules also predicts that permanent night workers and shift workers would be more likely to suffer from poor mental and physical health. The results of the present study support this notion: Permanent night workers perceive their general health status less often as (very) good compared to employees in general as well as compared to rotating night shift workers. The percentage of permanent night workers who rate their health status as (very) good slightly decreases with increasing weekly working hours but is relatively independent of weekend work.

Moreover, as opposed to employees in Germany in general, night workers more often report health complaints (see Figure 4). Not surprisingly, the percentage of people suffering from insomnia is notably higher among permanent night workers than among all employees. Figures for back pain or low back pain are also dramatically higher for permanent night workers. They also more often reported fatigue and exhaustion, depression, and physical exhaustion. Rotating night shift workers, however, suffered even more often from insomnia, fatigue and exhaustion as well as physical exhaustion. Thus, both permanent night workers and rotating night shift workers scored worse on all health indicators examined in the current study compared to employees in general.

Figure 4: Health Complaints of Permanent Night Workers, Rotating Night Shift Workers, and Employees in General

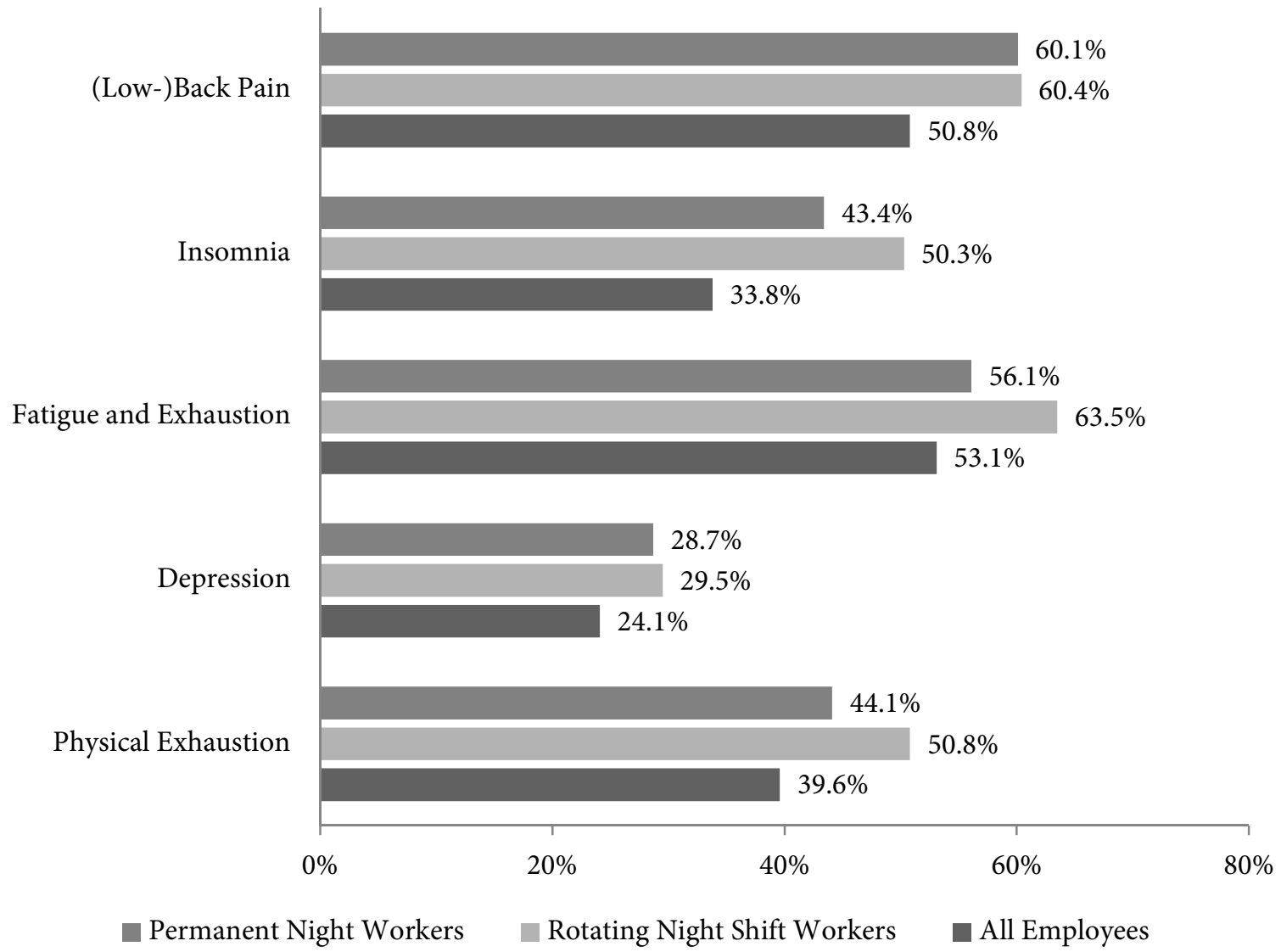




\section{Discussion}

The aim of the current study was to provide a holistic view of the under-researched group of permanent night workers using data from the representative BAuA-Working Time Survey 2015. While most studies do not distinguish between permanent night workers and those working in rotating shifts with night shifts, we focus explicitly on permanent night workers and compare these to rotating night shift workers as well as to employees in general. Thereby, we enhance understanding of this form of work and its associations with health and work-life balance.

Our findings show that just like in employees in general more permanent night workers are male than female - though not as many as among rotating night shift workers, have a medium level of education and predominantly work in healthcare, transport or food production. Permanent night workers more often work in services and less often in the public sector or industry compared to rotating night shift workers and employees in general. Further, we find that, compared to all dependent employees, night workers more often engage in work characterized by adverse physical and ambient working conditions as known from other representative German surveys, such as the BIBB/BAuA Employment Survey and the German Microcensus (Beermann 2010; Struck et al. 2012; Bundesanstalt für Arbeitsschutz und Arbeitsmedizin 2015). Nevertheless, some differences between permanent night workers and rotating night shift workers also become apparent. For instance, permanent night workers more often work in jobs where they have to lift and carry heavy loads or work under unfavorable lighting conditions, but they are less often exposed to noise. Thus, while permanent night work resembles rotating night shift work in some aspects, there are some noticeable differences between both kinds of night work.

Moreover, our study draws a detailed picture of the night shift and working time characteristics of permanent night workers. Half of permanent night workers have been working night shifts for more than five years. Thus, night work has been a part of their working life for a long period of time. In line with a standard workweek, it is most common for permanent night workers to work five consecutive night shifts. As night work is particularly common in workplaces that require twenty-four-seven staffing, weekend work is very widespread among permanent night workers. The average permanent night worker works 38.5 hours per week and the share of part time employees is higher among permanent night workers than among rotating night shift workers and employees in Germany in general. While permanent night workers are more often satisfied with their working hours than rotating night shift workers, satisfaction is lower than among all employees.

In line with the model by Tucker and Folkard (2012) on abnormal work schedules, compared to employees in Germany in general, permanent night workers and rotating night shift workers showed worse conditions on all health indicators as well as satisfaction with the work-life balance. Further, well-being tended to deteriorate in case of long working hours. Our analyses indicate that permanent night workers as well as rotating night shift workers face cumulative strains, both from the location of working hours as well as from further adverse work demands. These results demonstrate that permanent night workers as well as rotating night shift workers are highly stressed groups of employees, thereby confirming find- 
ings of earlier national and international studies (Alfredsson et al. 1991; Beermann 2010; Beutell 2010; Øyane et al. 2013; Angerer et al. 2017)

\section{Study Limitations and Avenues for Future Research}

Several limitations of the present study must be acknowledged. Since our results are based on cross-sectional descriptive analyses, we cannot draw causal conclusions regarding the role of permanent night work for health indicators or work-life balance. Most importantly, the current study does not allow inferences about the superiority or inferiority of permanent night work compared to rotational night shift work. In order to thoroughly analyzing the short- and long-term consequences of night work, experimental designs and longitudinal studies are required. First insights into the isolated effects of permanent night work could be obtained from matching procedures that compare permanent night workers with other employees with a similar socio-demographic background and comparable work characteristics (e.g., propensity score analysis).

A further limitation is that the investigated health indicators were not measured in physical examinations or diagnostic interviews but with questions screening for symptoms in telephone interviews. Moreover, due to the specific use of filters in the questionnaire, a post-hoc screening for permanent night workers was required and some questions were not administered to all permanent night workers. Hence, our results must be interpreted with caution.

Furthermore, there is a risk that the results of the present study are biased: Research shows that the adverse effects of working (time) conditions are generally underestimated because employees who feel that they cannot bear the strain do not apply for certain jobs. They also tend to leave the workforce or change into other jobs or to another working time schedule. Therefore, it is not unusual that employees remaining in shift work show a better health status than a comparison group of (e.g. day) workers - a phenomenon known as "healthy worker effect" (Li/Sung 1999). To improve our understanding of the employment histories of permanent night workers and possible strain-related job changes, future research should further investigate self-selection, adaptation, and attrition processes in the long run.

Finally, because of its national focus, our study may only speak for the German working population. We strongly encourage research on permanent night work in other countries to gather knowledge on specific national aspects and to develop a more global understanding of this kind of shiftwork.

\section{Implications for Practice}

Our results raise some concerns for the health status and work-life balance of night workers. If night work cannot be avoided, efforts should be made to create health-promoting boundary conditions. Therefore, we subscribe to the call made by Arlinghaus et al. (2016) who suggested combining night work with shorter working hours. This way, night workers would have more time for recovery and would be lured into night shift by financial incentives (e.g., shift premiums) to a lesser extent. Since our results also indicated that satisfaction with the work-life balance was lowest in case of long working hours, this might also benefit compatibility between work and private life. Although we found part-time work to be relatively common in 
our sample, an average of 38.5 actual weekly working hours reveals that there is still some room for reductions in working hours.

Further, it is likely that other adverse working conditions - especially physical and ambient environmental conditions - also contributed to health problems found among permanent night workers and rotating night shift workers. Also, previous research unveiled that the same job demands are more demanding at night than during the day (Arlinghaus et al. 2016). Therefore, to avoid such possibly harmful constellations, work demands should be reduced at night.

The frequent disturbances to the body clock and sleep associated with permanent night work and rotational night shift work are a challenge to the human body that may entail medical risks (Tucker/Folkard 2012). Moreover, the long-term consequences of working years or decades in permanent or rotating night shifts are not yet well understood. Thus, in line with several authors (Arlinghaus et al. 2016; Angerer et al. 2017) we suggest a closer medical supervision of night workers, especially if health restrictions are already present.

\section{Conclusion}

Overall, the present paper contributes to a more holistic picture of permanent night workers in Germany by presenting data on socio-demographic, structural, working time and job characteristics as well as associations with health and work-life balance. We hope to fuel research efforts as well as practical improvements that focus on this neglected but highly strained group of employees.

\section{References}

Alfredsson, Lars, Torbjörn Åkerstedt, Malin Mattsson and B. Wilborg (1991). Self-reported health and well-being amongst night security guards: a comparison with the working population, Ergonomics, 34, 525-530.

Angerer, Peter, Renate Schmook, Irina Elfantel and Jian Li (2017). Night work and the risk of depression: A systematic review, Deutsches Ärzteblatt International, 114, 404-411.

Arlinghaus, Anna, Johannes Gärtner, Sylvia Rabstein, Sebastian Schief and Céline Vetter (2016). Dauernachtarbeit-Eine Sichtung des vorhandenen Wissenstandes mit Thesen, Empfehlungen \& Forschungsfragen, SOZIALPOLITIK.CH, 2, 1-11.

Beermann, Beate (2010). Nacht-und Schichtarbeit. In: Fehlzeiten-Report 2009: Arbeit und Psyche: Belastungen reduzieren - Wohlbefinden fördern. Heidelberg: Springer.

Beutell, Nicholas J. (2010). Work schedule, work schedule control and satisfaction in relation to workfamily conflict, work-family synergy, and domain satisfaction, Career Development International, $15,501-518$.

Bundesanstalt für Arbeitsschutz und Arbeitsmedizin (2015). Brennpunkt Nachtarbeit - Häufige Arbeitsbelastungen immer noch aktuell. In: BIBB/BAuA-Faktenblatt. Dortmund: Bundesanstalt für Arbeitsschutz und Arbeitsmedizin. 
Bundesanstalt für Arbeitsschutz und Arbeitsmedizin (2017). Sicherheit und Gesundheit bei der Arbeit: Unfallverhütungsbericht Arbeit - 2016. Dortmund: Bundesanstalt für Arbeitsschutz und Arbeitsmedizin.

Bundesministerium für Arbeit und Soziales (2017). Das Arbeitszeitgesetz. Bonn: Bundesministerium für Arbeit und Soziales.

Czeisler, Charles A. and Derk-Jan Dijk (1995). Use of bright light to treat maladaptation to night shift work and circadian rhythm sleep disorders, Journal of Sleep Research, 4, 70-73.

Eastman, Charmane I., Karen T. Stewart, Michael P. Mahoney, Liwen Liu and Louis F. Fogg (1994). Dark goggles and bright light improve circadian rhythm adaptation to night-shift work, Sleep, 17, 535-543.

Eurofound (2017). Working time patterns for sustainable work. Luxembourg: Publications Office of the European Union.

Folkard, Simon (2008). Do permanent night workers show circadian adjustment? A review based on the endogenous melatonin rhythm, Chronobiology International, 25, 215-224.

German Federal Statistical Office (2008). Gliederung der Klassifikation der Wirtschaftszweige. Wiesbaden: German Federal Statistical Office.

Häring, Armando, Holger Schütz, Reiner Gilberg, Martin Kleudgen, Anne M. Wöhrmann and Frank Brenscheidt (2016). Methodenbericht und Fragebogen zur BAuA-Arbeitszeitbefragung 2015. Dortmund: Bundesanstalt für Arbeitsschutz und Arbeitsmedizin.

Hellert, Ulrike (2014). Arbeitszeitmodelle der Zukunft-inkl. Arbeitshilfen online: Arbeitszeiten flexibel und attraktiv gestalten. Freiburg: Haufe-Lexware.

International Labour Office (2012). International Standard Classification of Occupations: ISCO-08. Geneva: International Labour Office.

Knutsson, Anders and Anders Kempe (2014). Shift work and diabetes-a systematic review, Chronobiology International, 31, 1146-1151.

Li, Chung Yi and F-C Sung (1999). A review of the healthy worker effect in occupational epidemiology, Occupational Medicine, 49, 225-229.

Liersch, Anja (2014). Arbeitsunfälle und arbeitsbedingte Gesundheitsprobleme, Wirtschaft und Statistik (Sept), 561-574.

Øyane, Nicolas M. F., Ståle Pallesen, Bente E. Moen, Torbjörn Åkerstedt and Bjørn Bjorvatn (2013). Associations between night work and anxiety, depression, insomnia, sleepiness and fatigue in a sample of Norwegian nurses, PloS one, 8, e70228.

Pfahl, Svenja, Dietmar Hobler and Sandra Horvath (2017). Abend- und Nachtarbeit von Abhängig Beschäftigten 1996 - 2015. In: WSI GenderDatenPortal. Düsseldorf: Hans-Böckler-Stiftung.

Puch, Katharina (2010). Arbeiten, wenn andere schlafen. In: STATmagazin. Wiesbaden: German Federal Statistical Office.

Ramin, Cody, Elizabeth E. Devore, Weike Wang, Jeffrey Pierre-Paul, Lani R. Wegrzyn and Eva S. Schernhammer (2015). Night shift work at specific age ranges and chronic disease risk factors, Occupational and Environmental Medicine, 72, 100-107. 
Struck, Olaf, Matthias Dütsch, Verena Liebig and Angelina Springer (2012). Arbeit zur falschen Zeit am falschen Platz? Eine Matching-Analyse zu gesundheitlichen Beanspruchungen bei Schicht-und Nachtarbeit, Journal for Labour Market Research, 47, 245-272.

Tucker, Philip and Simon Folkard (2012). Working time, health and safety: A research synthesis paper. In: ILO Working Papers. Geneva: International Labour Organization.

UNESCO Institute for Statistics (2012). International standard classification of education: ISCED 2011. Montreal: UNESCO Institute for Statistics Montreal.

Wöhrmann, Anne M., Susanne Gerstenberg, Lena Hünefeld, Franziska Pundt, Anna Reeske-Behrens, Frank Brenscheidt and Beate Beermann (2016). Arbeitszeitreport Deutschland 2016. Dortmund: Bundesanstalt für Arbeitsschutz und Arbeitsmedizin. 